\title{
Tourisme et politique du livre et de la lecture en Bretagne : enjeux politiques d'une construction identitaire régionale
}

Mathilde Sempé

\section{OpenEdition}

Journals

Édition électronique

URL : http://journals.openedition.org/tourisme/413

DOI : $10.4000 /$ tourisme.413

ISSN : 2492-7503

Éditeur

Éditions touristiques européennes

Édition imprimée

Date de publication : 1 juin 2012

Pagination : 47-61

ISSN : 2109-5671

\section{Référence électronique}

Mathilde Sempé, «Tourisme et politique du livre et de la lecture en Bretagne : enjeux politiques d'une construction identitaire régionale », Mondes du Tourisme [En ligne], 5 | 2012, mis en ligne le 30 septembre 2015, consulté le 23 avril 2019. URL : http://journals.openedition.org/tourisme/413 ; DOI 10.4000/tourisme.413

\section{(c) (1) $\odot$

Mondes du tourisme est mis à disposition selon les termes de la licence Creative Commons Attribution - Pas d'Utilisation Commerciale - Pas de Modification 4.0 International. 


\title{
Tourisme et politique du livre
}

\author{
et de la lecture en Bretagne :
}

\section{enjeux politiques}

\section{d'une construction identitaire régionale}

\author{
Mathulde Sempé
} [mathildesempe@yahoo.fr] Attachée temporaire d'enseignements et de recherches (Ater) en science politique • Groupe d'analyse politique

Résumé. Le processus de décentralisation culturelle en marche dès les années 1970 a opéré un transfert de compétences de l'État vers les collectivités territoriales et renforcé les mécanismes de concurrence et de distinction dans la course à l' "identité" culturelle locale et régionale. Dès lors, le "tourisme", envisagé au prisme d'une nouvelle catégorie d'intervention de l'action publique - "la politique culturelle" -, n'apparaît plus uniquement comme une ressource économique mais également comme une ressource politique mettant en évidence les stratégies régionales de réification d'une histoire et d'un territoire spécifiques. Le regard porté sur la sociogenèse d'une politique du livre et de la lecture en Bretagne - comme focale de l'analyse d'enjeux de luttes constitués, pour un ensemble d'acteurs hétérogènes, autour d'un objet culturel - tend à révéler le travail d'homogénéisation et d'imposition de représentations d'une identité culturelle ; et également à retracer le processus d'institutionnalisation des catégories de "tourisme" et de "région".

Abstract. The process of cultural decentralization started in the 70's, and carried out a transfer of competences from the State to the territorial collectivities which reinforced the competition and distinctive mechanisms in the running for local and regional cultural identity. Thereby, "tourism" is considered as a new category of intervention within the public action: "the cultural policy"; and no longer only as an economic resource, but also a political one, highlighting the regional strategies creating a reification of a history and a specific territory. The social genesis in regards of the policy of the book and reading in Brittany - as support of the analysis of struggling issues constituted, for a group of heterogeneous protagonists, around a cultural object - tends to reveal the process of homogenization and imposition of cultural identity's representations; which can also be retraced to the institutionalized process of categories within "tourism" and off the "region". 


\section{6 e tourisme s'entend [pour la région Bretagne] comme} activité essentielle à la vie des nations, en raison de ses effets directs sur les secteurs socianx, culturels, éducatifs et économiques des sociétés nationales et sur leurs relations internationales partout dans le monde ${ }^{\text {(1) }}$. Les enjeux de luttes définitionnels constitués autour du "tourisme" contribuent au flou de cette notion. Le tourisme est-il une ressource économique constitutive d'enjeux politiques ? Est-il une variable influant sur la pratique culturelle ou, au contraire, est-il un concept écran nous détournant des mécanismes et comportements à l'œuvre dans l'action publique ? Il convient par conséquent d'appréhender le tourisme (ou "les" tourismes) dans sa pluralité compte tenu des usages différenciés dont il fait l'objet. Le "tourisme", tel que le sens commun le fait apparaître dans son acception de loisirs, lié aux congés payés, aux vacances et aux voyages, ou encore aux déplacements - que les critères de "durée" et de "motif du séjour" caractérisent officiellement (Stock, 2003, p. 10) (2) -, se réalise dans la production de nouvelles catégories morales de perception. Aussi, il peut être compris comme un ensemble de représentations que l'on se fait de pratiques (de déplacements d'individus), véhiculées par les acteurs de la fabrication de politiques publiques et reproduites (dans leurs déplacements effectifs) par leurs usagers, autrement dit "les touristes".
L'histoire du tourisme a partie liée avec l'apparition des "régions", que l'on appréhendera comme un espace géographique d'observation de pratiques touristiques, notamment dans l'analyse du processus de construction sociale du politique. La genèse d'une institution est révélatrice en ce sens qu'elle laisse entrevoir les enjeux de luttes pour la définition légitime de la région, à laquelle participent un ensemble d'acteurs hétérogènes - auxquels s'ajoute le chercheur dès lors qu'il nomme et objective cette catégorie. "Tout énoncé sur la région fonctionne comme un argument qui contribue, et d'autant plus largement qu'elle est plus largement reconnue, à favoriser ou à défavoriser l'accès de la région à la reconnaissance et, par là, à l'existence" (Bourdieu, 1980, p. 68). La "région” apparaît alors comme le résultat de représentations spontanées contre lesquelles les acteurs locaux allaient revendiquer des singularités régionales. Le processus de déconstruction des évidences et de redéfinition "légitime" de la Bretagne, dont il sera question ici, s'établit au fil des mouvements sociaux "régionalistes", des années 1950 aux années 1970, avec l'apparition de la question culturelle comme enjeu de luttes sociales. Les revendications des groupes sociaux mobilisés sont alors réinvesties par les acteurs de l'espace politique régional, qui achèvent de transformer un enjeu culturel en un enjeu politique. Cependant, l'intégration d'un “impératif culturel" ${ }^{(3)}$ ne peut se comprendre qu'au regard de l'imposition de catégories de pensée de l'État. Ainsi, la "question régionale" et la "démocratisation de la culture" apparaissent dans les années 1960, sous le ministère Malraux, et se poursuivent dans les années 1980, sous le ministère Lang. Il s'agit par conséquent de rendre compte du processus de décentralisation qui se dessine progressivement et s'institutionnalise dans les années 1980 pour conférer des compétences plus larges aux collectivités locales et donner naissance à un nouvel espace politique : la région.

La régionalisation ou production symbolique de la région révèle "l'illusion identitaire” (Bayart, 1996), et l'identité - entendue comme la construction d'un imaginaire permettant à la fois la fabrication et l'homogénéisation des représentations sur la région mais également la distinction des autres collectivités territoriales - apparaît comme une ressource culturelle essentielle à la formation des aménagements touristiques $^{(4)}$. La construction d'une identité suppose l'imposition d'une vision du monde par les professionnels de la politique tout comme l'adhésion à ces représentations par les agents sociaux. Aussi est-elle le résultat d'une objectivation du territoire rendant possibles l'identification et la distinction par la réécriture des histoires locale et régionale, cellesci étant prises dans les enjeux de luttes du présent pour la définition légitime de l'identité culturelle régionale. On considérera, à la suite de Pierre Bourdieu, Roger Chartier et Robert Darnton, la culture comme 
"un univers symbolique", le symbole étant entendu comme vecteur de représentations (Bourdieu et al., 1985, p. 9l). Une politique culturelle doit être envisagée comme l'ensemble des usages différenciés par les professionnels de la politique de symboles (que sont, ici, l'histoire réifiée et l'identité culturelle imaginée) œuvrant à l'imposition ou à la conservation de représentations se traduisant dans des pratiques et des croyances tendant à assurer la pérennité des institutions. De ce point de vue, la revendication d'une culture constitutive a priori d'une identité particulière s'envisage concurremment aux autres. La production d'une culture commune, ou plus exactement de la croyance en une culture commune, revient au travail d'imposition et d'homogénéisation des représentations, donc à "l'intériorisation de l'arbitraire culturel" (Bourdieu et Darbel, 1969, p. 162) réalisant par là même l'unité. La construction d'un arbitraire culturel passe par le travail symbolique des agents sociaux dominants que sont les acteurs des champs intellectuel, médiatique et politique dans l'affirmation de nouvelles institutions régionales. Décrypter cet arbitraire, c'est s'interroger sur les conditions de possibilités sociales et historiques de production d'une politique culturelle : la politique du livre et de la lecture en Bretagne. En quoi la politique du livre - dans ses usages touristiques - constitue-t-elle un enjeu politique de construction d'une identité culturelle régionale ?

Il convient, dans une perspective socio-historique, de replacer les agents sociaux dans des trajectoires particulières, observables dans un réseau de relations sociales et comprises dans un contexte local, régional et national de production des politiques du livre et de construction sociale d'une identité culturelle. La sociogenèse de cette construction identitaire régionale permettra d'aboutir à la politique du livre comme englobant la perspective touristique dans le processus de fabrication de cette identité régionale. Notre démarche entend interroger un système de rapports de force entre des acteurs aux intérêts divergents s'attachant néanmoins à la promotion d'une identité culturelle bretonne et, plus largement, à la construction d'une légitimité politique régionale. Nous procéderons, dans le premier temps de ce travail, au retour sur l'histoire d'une double construction. La première construction est celle de la catégorie de région et, avec elle, d'institutions régionales tenues de conduire cette seconde construction qu'est la naissance d'une politique culturelle en partie orientée vers le ou les tourismes. Dans un second temps, nous aborderons l'analyse de ce secteur régional du livre, comprenant le tourisme comme un élément essentiel de développement économique (et culturel) de la région, au prisme des échanges de coups entre des acteurs institutionnels et des acteurs privés, entre des collectivités territoriales qui entrent en compétition afin d'institutionnaliser des initiatives liées au livre.

\section{DES “RÉGiOnALISMES" \\ À LA RÉGIONALISATION : TOURISME ET CONSTRUCTION D’UNE IDENTITÉ RÉGIONALE}

Procéder à la sociogenèse de la région, c'est rendre compte des rapports de force qui se jouent dans la lutte pour une identité culturelle régionale. Le retour opéré sur ce processus de régionalisation nous porte à nous intéresser dans un premier temps aux mouvements sociaux dits "régionalistes" et à leur opposition au "tourisme" dans la construction sociale d'une représentation de la région. Le second temps de cette institutionnalisation s'observe dans des stratégies d'appropriation et de redéfinition, par les institutions régionales, de cette identité fabriquée. Stratégies objectivables dans la mise en œuvre d'une politique culturelle imaginée autour du tourisme.

\section{Sociogenèse de la construction d'une identité régionale : les “régionalismes" contre le tourisme}

L'analyse des enjeux politiques et touristiques du livre en région nécessite des précisions sur l'espace étudié. Toutefois, le flou qui entoure la catégorie de région renvoie à la difficulté de la définir comme un niveau de décentralisation, un espace ou un groupe social. La polysémie du terme suppose un travail d'homogénéisation des représentations produites sur la région. Ainsi la fabrication d'une identité régionale révèle-t-elle ce travail de construction 
et d'homogénéisation de collectifs hétérogènes. Par conséquent, "l'identité culturelle" régionale comme "allant de soi" dissimule le travail d'objectivation de celle-ci par des acteurs des champs politique et intellectuel mais également par des groupes sociaux mobilisés. L'intériorisation du rapport de domination étant rendue possible par ce processus inconscient d'incorporation, par les dominés (et les dominants), de représentations socialement constituées... C'est ce que Pierre-Jean Simon relève dans "la bretonnité plus ou moins imposée aux Bretons et qu'ils ont plus ou moins intériorisée" (Simon, 1999, p. 49). Les conditions sociales et historiques de possibilité de cette identité culturelle se comprennent dès lors dans les luttes symboliques pour la définition et la construction d'un espace politique régional. Ces rapports de force apparaissent, dans le premier temps du processus de régionalisation, au travers des revendications régionales, que nous ne pouvons saisir qu'au regard de leur réintroduction dans un contexte national de production de celles-ci. L'on assiste, à partir des années 1950, à une transformation des représentations et des pratiques sociales jusqu'alors instituées ; la conjoncture de crise politique de mai-juin 1968 prenant en partie sens dans cette histoire des mouvements sociaux qui s'inscrivent dans un processus de déconstruction des évidences. "Le mouvement critique de maijuin 68 travaille à délégitimer ce qui se présentait et était perçu aupara- vant comme légitime. [...] Mai 68 emporte donc avec lui un travail multiforme, et pas toujours conscient, de légitimation de l'illégitime" (Damamme, Gobille, Matonti et Pudal, 2008, pp. 2I-22). Les revendications d'identités culturelles observées dans les mouvements sociaux régionalistes des années 1960 aux années 1980 - dissimulent en réalité des revendications politiques qui favorisent de nouvelles pratiques et croyances contribuant à l'autonomisation des institutions régionales et à la structuration d'un nouvel espace politique régional (Pasquier, 2004). Les mobilisations constituées autour du "droit à l'autodétermination"(5) entrent en contradiction avec le processus de renforcement de l'État moderne centralisé. C'est pourquoi les mouvements régionalistes bretons se construisent en opposition à la définition jusqu'alors légitime de l'identité bretonne, celle que véhiculent les voyages touristiques et qui perdure jusque dans les années 1950-1960. Stendhal, dans ses mémoires d'un touriste en Bretagne écrivait : " $\mathrm{La}$ partie de la Bretagne où l'on parle breton, d'Hennebont à Josselin et à la mer, vit de galettes de farine de sarrasin, boit du cidre et se tient absolument aux ordres du curé" (Stendhal, 1984, p. II [récit paru en 1838]). L'image de la Bretagne incarnée par la domestique bretonne arrivée à Paris (connue sous les traits de Bécassine) reproduit par là même les représentations liées à la nonmaîtrise de la langue française (Bachimon, 2006) et réactualise les stéréotypes d'un folklore et d'une culture rurale bretonne (Bertho, 1980).

"La réalité s'imposait dans toute son horreur : nous n'étions que des ploucs, des paysans, des bouseux, des préhistoriques, des retardés, des oubliés de la civilisation, des sauvages. Des minables. Nous jouions les fiers-à-bras et depuis le début, sans le savoir, nous avions une pancarte 'je suis un imbécile' accrochée dans le dos. À mourir de honte" (Le Bris, 1977, p. 27).

"Ainsi l'esclave se moque-t-il des travers de son maître - il n'en reste pas moins esclave. Bien sûr, ils [les touristes] n'étaient pas nos maîtres, nous n'étions pas leurs esclaves du moins pas encore - mais ils nous humiliaient déjà un peu [...]. Comment donc avions-nous pu oublier cette grande leçon que tout ce qui vient de l'extérieur est nécessairement mauvais ? [...] ' $I l s$ ' venaient d'ailleurs, leur pays ne pouvait donc être, comme dit la chanson, que Paris. [...] Tout le monde [dans le Trégor] sait qu'un touriste, cela vient de Paris - d'ailleurs nous disions indifféremment 'touriste' ou 'Parisien'. [...] Si votre Paris est un paradis, que venez-vous donc chercher, dans notre pouilleuse Bretagne, que vous n'avez pas ?” (Le Bris, 1977 , pp. 22-34).

L'on assiste progressivement à la remise en cause de ce qui avait été intériorisé par les agents sociaux comme des représentations négatives de la Bretagne et d'eux-mêmes. L'incorporation de leur propre indignité sociale fait l'objet, pour de 
nombreux agents sociaux chez qui le processus de politisation avait opéré, d'un désenchantement visant à mettre en doute les représentations socialement instituées. La politisation est entendue ici comme l'incorporation de nouvelles façons de penser, de nouvelles logiques politiques. Elle est ce processus de socialisation politique intervenu chez l'individu, ayant provoqué son intérêt pour la politique (Weber, 1983; Braud, 2008). L'enjeu consiste, pour les groupes sociaux mobilisés, à délégitimer la vision dominante de la Bretagne afin de légitimer et d'imposer une nouvelle définition de la région. Les revendications d'une "culture populaire" ${ }^{(6)}$ distincte des instances et pratiques culturelles légitimes et légitimées s'observent dans des formes d'engagement différenciées, regroupées sous cet ensemble hétérogène qu'est l'Emsav (le mouvement culturel breton). Par ailleurs, l'investissement de groupes sociaux dans la défense de l'environnement et contre le nucléaire notamment avec les grandes mobilisations autour de la marée noire de l'Amoco Cadiz, en 1978, et de la centrale nucléaire de Plogoff, en 1979 - s'explique dans la compréhension du tourisme comme la cause de ce qui "détruirait la nature (le 'paysage') et les sociétés qui l'accueillent” (Stock, 2003, p. 4) et, plus largement, dans une dynamique des années 1970 de remise en cause de l'ordre symbolique établi.

"Cela relève de la même problématique de conservation à la fois de l'identité bretonne qui est consti- tuée par la langue, la culture, les traditions revisitées et modernisées et puis des questions d'environnement" (entretien avec une élue au conseil régional de Bretagne, 9 février 2012).

Au cours des années 1960-1970, les mouvements culturels bretons apparaissent, avant l'appropriation idéologique par les institutions régionales, comme les structures historiquement légitimes de production d'une identité régionale (Fournis, 2006). Celle-ci est envisagée en opposition à ce qui provient de l'extérieur, l'extérieur étant souvent associé à "Paris" et aux croyances véhiculées par les voyages touristiques en Bretagne. Les luttes symboliques pour la définition légitime de la Bretagne traduisent ainsi le rapport de domination culturelle institué entre "la région" et "Paris". "Si la région n'existait pas comme espace stigmatisé, comme 'province' définie par la distance économique et sociale (et non géographique) au 'centre', c'est-à-dire par la privation du capital (matériel et symbolique) que concentre la capitale, elle n'aurait pas à revendiquer l'existence: c'est parce qu'elle existe comme unité négativement définie par la domination symbolique et économique que certains de ceux qui en participent peuvent être conduits à lutter [...] pour en changer la définition" (Bourdieu, 1980, p. 70). C'est pourquoi la construction d'une identité culturelle bretonne tend a priori à exclure les pratiques et représentations touristiques pour envisager par la transformation de probléma- tiques culturelles en enjeux politiques - le processus de naturalisation de la région.

Les revendications culturelles bretonnes s'appuient sur des critères supposés objectifs de l'identité régionale tels que la langue, qui apparaît dès lors comme le résultat d'une objectivation la rendant "évidente" dans son association à l'idée de région (Bourdieu, 1980 ; Thiesse, 1999). La systématisation des rapports de force constitués autour de la langue vise une reconnaissance symbolique d'une "culture régionale" significative de la région. Lorsque les associations de soutien à la langue bretonne évoquent l'hypothèse d'une charte culturelle de Bretagne, en guise de réponse au discours de Valéry Giscard d'Estaing prononcé en 1977 à Ploërmel ${ }^{(7)}$, la langue bretonne devient alors l'enjeu de luttes d'identification régionale entre les groupes sociaux mobilisés et ces nouvelles institutions désormais représentatives de la région que sont le conseil régional et le comité économique et social de Bretagne. Les usages différenciés de la langue bretonne révèlent l'inscription des acteurs territoriaux dans la lutte pour la détermination du contenu de la charte, son appropriation permettant la transformation des positions occupées par les agents sociaux et, par là même, la redéfinition légitime de la politique culturelle régionale.

"La présente Charte constitue [...] un acte de reconnaissance de la personnalité culturelle de la Bretagne et de l'engagement d'en 
garantir le libre épanouissement. Elle répond au désir de respect de leur identité exprimé par la population bretonne et ses élus. Elle constitue un pas vers la prise en charge, par la région, de sa politique culturelle. Elle se place ainsi dans le sens d'une évolution générale des États favorable aux personnalités régionales. La personnalité culturelle de la Bretagne s'est toujours manifestée de manière originale dans la totalité des aspects de la vie sociale. [...] La langue bretonne en est une de ses composantes fondamentales. Cependant la culture en Bretagne ne peut se réduire à une expression traditionnelle ni à une culture populaire d'essence rurale. Il ne saurait exister de culture bretonne authentique que résolument ouverte vers l'extérieur et sur l'avenir. Le propos de la présente Charte est de mettre fin au divorce qui existait entre une culture régionale, toujours riche et vivante, mais qui risquait de se replier sur elle-même, et une culture dominante qui l'ignorait trop souvent" (préambule de la Charte culturelle de Bretagne $)^{(8)}$.

La construction d'une identité culturelle devient nécessairement politique en ce sens que la question culturelle, saisie par des groupes sociaux mobilisés, est réappropriée par des institutions locales et régionales, faisant ainsi de l'enjeu culturel un enjeu politique. L'usage de la "culture bretonne" participe aux stratégies de présentation de soi des acteurs de l'espace politique régional se positionnant dès lors comme les nouveaux "porte-parole du mouvement culturel breton" (Pasquier, 2004, p. 103).

\section{L'institutionnalisation \\ de la région et \\ les enjeux touristiques \\ d'une politique culturelle}

La loi du 5 juillet 1972 permet la création d'établissements publics régionaux et, en leur sein, des structures que sont le conseil régional et le comité économique et social. Le processus de régionalisation ou l'institutionnalisation de la région s'observe dans la lutte qui se joue pour l'acquisition, par ces instances, d'un pouvoir symbolique, celui de l'imposition d'une vision légitime de la région. La légitimation de cette institution s'opère notamment en 1982, lorsque la région devient une collectivité territoriale ; également en 2002, lorsque le processus de décentralisation administrative entraîne à nouveau un transfert de compétences de l'État vers les collectivités territoriales. Plus que l'acquisition de nouvelles prérogatives, il s'agit d'une accélération du processus de politisation des acteurs territoriaux et des institutions régionales. Lors des élections de 1986, un nouveau type de professionnels de la politique apparait. Ce composite hétérogène tend à se distinguer des élections "routinisées" dans sa détermination sociologique d'accès à la position d'élu ${ }^{(9)}$. Les femmes, les jeunes et les professions intermédiaires, habituellement sous-représentés parmi les professionnels de la politique, entrent désormais dans le jeu politique. La composition sociologique des structures régionales s'explique par la création d'une nouvelle institution qui n'est pas encore "routinisée" dans ses pratiques et ses croyances, ces dernières entraînant a priori la survie et la légitimation de l'institution régionale. Il semblerait par conséquent que l'enjeu politique des élections régionales apparaisse, pour les professionnels de la politique qui dominent alors dans l'espace régional, comme moins déterminant que les élections municipales (Alliès, 1989).

L'incorporation par les agents sociaux de nouvelles façons de faire et de penser se traduit par des transformations propres aux structures institutionnelles. Le processus de professionnalisation induit par les logiques du champ politique - dont la propriété principale reste l'imposition ou la conservation d'une vision légitime du monde social - suppose dès lors des rapports de force entre les collectivités territoriales tant au niveau local que régional. Il s'agit de se distinguer des autres régions et, au sein de cet espace, entre les différentes localités. La création de plusieurs niveaux de décentralisation accentue cette compétition politique engendrant simultanément des "modèles d'action publique" (Arnaud, Le Bart et Pasquier, 2006, p. 16) ; ceux-ci participant de cette imposition de représentations et rendant compte de l'illusion d'une homogénéité culturelle, de l'illusion d'une identité régionale. Aussi les acteurs locaux et régionaux s'emploient-ils à la construction d'une image culturelle de la Bretagne dis- 
tincte de celle des autres régions et se traduisant dans cette catégorie de l'action publique qu'est la politique culturelle. Ces luttes structurées autour d'enjeux culturels déterminés par un contexte d'accélération du processus touristique sont dès lors envisagées au regard d'une compétition interrégionale ayant pour fin le développement économique. L'identité, socialement construite, s'appuie sur un passé réinventé tenant compte des représentations spontanées, imposées autrefois par une vision dominante véhiculée notamment par les touristes euxmêmes. De ce point de vue, la variable "tourisme" permet la fabrication des identités locales au travers de l'usage d'une histoire particulière, en partie réifiée et imaginée en fonction des contraintes qu'impose le développement économique. " $\mathrm{La}$ 'spécificité' du territoire régional tient à l'appropriation et à la 'valorisation' [par les agents sociaux] d'un patrimoine historique, afin de structurer une identité culturelle vers les habitants (logique d'identification) et vers l'extérieur (logique d'attraction touristique). Dans les deux cas, l'affirmation d'une identité territoriale se fonde sur des politiques publiques" (Hassenteufel et Smith, 2002, p. 68).

"Le tourisme est devenu une activité économique et, en tant que telle, se voit de plus en plus lié aux différentes politiques de développement. [...] Toutes ces formes de tourisme se développent principalement à travers l'exploitation des ressources naturelles, historiques et culturelles.
La Bretagne occupe une position relativement privilégiée sur le marché $d u$ tourisme en raison de son histoire et des beautés naturelles dont elle dispose. Depuis longtemps considérée comme une destination touristique, [...] elle n'a eu de cesse, depuis, de renforcer cette vocation" ${ }^{\prime(10)}$.

La normalisation d'une catégorie de l'action publique nous contraint à envisager désormais la politique culturelle dans les enjeux économiques qu'elle induit. C'est pourquoi les offres culturelles tendent à répondre à une demande touristique capable de produire des bénéfices économiques déterminants dans l'entretien de la compétition politique entre les collectivités territoriales. La distinction s'opère dans les usages différenciés de "l'histoire" permettant, au travers de critères "objectifs" de l'identité régionale, de rendre compte d'une spécificité régionale. La création de labels, agréés par l'État, tels que "Ville d'art et d'histoire" (obtenu par l'entretien ou la restauration d'un riche patrimoine historique et culturel) $)^{(11)}$, "Station classée" (permettant la reconnaissance officielle de villes à vocation touristique) ou "Petites Cités de caractère", s'inscrit dans un processus d'identification territoriale - le classement opéré par la région selon l'attribution de ces labels contribuant de fait à la confirmation des représentations produites sur les villes touristiques. La distinction établie entre les différentes collectivités territoriales s'emploie d'une part à entretenir la concurrence entre les localités, d'autre part à renforcer la reconnaissance symbolique de la région.

L'imposition du "tourisme" comme catégorie au prisme de laquelle s'envisage désormais une politique culturelle participe du processus d'homogénéisation des représentations construites et reproduites sur la Bretagne ; et la fabrication d'une identité culturelle spécifique passe notamment par l'usage touristique du livre, dans la mesure où celui-ci contribue au développement économique de la région. Ainsi la politique du livre et de la lecture figure-t-elle parmi les stratégies de distinction culturelle du conseil régional. Le travail de représentation politique tendant à homogénéiser des croyances passe par la création, en 2008, d'une structure régionale pour "le livre" en Bretagne : "Livre et lecture en Bretagne". Ces structures culturelles sont nées de l'association entre les agences régionales de coopération entre bibliothèques $(\mathrm{COBB})$ et les centres régionaux du livre (CRL), qui mènent des actions de soutien à la création et à l'édition. Cet établissement public de coopération culturelle (EPCC), réunion du livre et de la lecture publique, est né de l'association entre les centres régionaux du livre (CRL), qui mènent des actions de soutien à la création et à l'édition, et les agences régionales de coopération entre bibliothèques (COBB). Le CRL Bretagne a été créé en 1992 à Quimper par un ensemble d'acteurs issus du champ associatif; ces acteurs ayant un poids considérable 
dans les rapports de force engagés autour des enjeux politiques (et touristiques) que pose "le livre" dans sa configuration régionale. Toutefois, la constitution d'une politique culturelle régionale, vitrine du travail d'homogénéisation des images produites sur la Bretagne, résulte précisément de ce rapport de force constitué entre des structures culturelles ${ }^{(12)}$, anciennement créées par des groupes sociaux mobilisés autour de la "culture bretonne", et les institutions administratives que sont le conseil économique, social et environnemental (CESER) - qui a remplacé en 1992 le comité économique et social et que nous nommons ici, par souci de concision, "conseil économique et social" - et, principalement, le conseil régional de Bretagne. "L'institution (au sens général) résulte d'abord d'un effet de spécification par lequel elle s'assure un territoire d'intervention. [...] Elle s'applique à conquérir un territoire géographique, matériel ou symbolique aux dépens d'autres structures plus anciennes" (Dauvin, 1993, p. 17). L'acquisition du pouvoir de redéfinition d'une identité territoriale par le conseil régional de Bretagne s'observe pratiquement dans la réappropriation de ces structures culturelles constituées autour du livre. L'intérêt pour le conseil régional de Bretagne apparaitt tant économique que culturel.

"La région a soubaité que ce soit un espace propice au commerce, ce qui est essentiel en ce lieu, et indispensable aux éditeurs qui font l'effort à la fois financier et personnel de se déplacer ici. [...] Il s'agit donc cette année d'un tournant dans l'intérêt que porte la région au livre, c'est un intérêt aussi économique que culturel. [...] Il y a bien une industrie du livre en Bretagne et la région entend la défendre avec autant d'énergie qu'elle défend son identité et sa culture" (Note du 20 mars 2002 de la directrice déléguée à la culture au conseil régional de Bretagne, à l'attention du président du conseil régional $)^{(13)}$.

Livre et lecture en Bretagne est transféré en 2002, sous l'impulsion de la région, à Rennes, alors capitale administrative et, par le déplacement de la structure, "capitale culturelle" de la Bretagne. La réappropriation, par les institutions régionales, de structures culturelles auparavant créées par des associations, contribue à la redéfinition "légitime" de la politique du livre et de la lecture en Bretagne, plus encore à l'institutionnalisation de l'idée de région.

\section{“LE LIVRE” COMME ENJEU DE LUTTES TOURISTIQUES ENTRE COLLECTIVITÉS LOCALES}

L'analyse d'une politique publique locale implique de s'intéresser aux stratégies et aux échanges de coups qui permettent de retracer le processus de fabrication de celle-ci. Il s'agit de mettre en évidence les propositions culturelles des associations et les transformations de ces structures dans leur réappropriation par les acteurs du champ politique local. Les rapports de force engagés pour la définition et les usages légitimes du livre au sein d'une ville nous conduisent par conséquent à envisager "le livre" dans les enjeux politiques qu'il induit. En quoi "le livre", entendu a priori comme une œuvre littéraire, peut-il être saisi par des institutions locales au point d'être appréhendé comme un produit commercial œuvrant au développement touristique d'une municipalité ?

\section{Les enjeux touristiques \\ d'une politique du livre : \\ l'exemple de Saint-Malo}

La politique du livre de la ville de Saint-Malo est particulièrement intéressante du point de vue de la professionnalisation de structures associatives que sont Étonnants Voyageurs (festival du livre de voyage) et Quai des Bulles (festival de la bande dessinée) et, plus largement, de la constitution d'un espace d'analyse des enjeux touristiques que pose "le livre". La genèse de ces manifestations se comprend dans un espace relationnel plus large, où "le livre" procède d'une stratégie municipale comprenant "l'événementiel" comme un élément essentiel dans l'élaboration d'une politique culturelle. Elle s'inscrit dans une histoire "spécifique" et ce, afin de contribuer à l'invention d'une identité locale construite en distinction des autres localités. Dès lors, la Route du Rhum (course nautique transatlantique), la Route du Rock (festival de musique) tout comme les Étonnants Voyageurs procèdent conjointement d'un rattachement à une histoire locale, celle des corsaires malouins, du voyage, de la mer... : 
l'identification de la ville est ainsi facilitée - au-delà du travail constitué autour de l'imaginaire - par l'industrie touristique suscitée par ces investissements opérés sur le "culturel”. La manifestation littéraire constitue moins un enjeu culturel que commercial, et en particulier touristique, dès lors qu'une grande partie du chiffre d'affaires des librairies malouines est réalisée durant le festival Étonnants Voyageurs ${ }^{(14)}$, luimême organisé sur un week-end prolongé du mois de mai et dont dépendent les activités hôtelières et de restauration.

“Quand on regarde bien ce qu'est Saint-Malo, ce qui est mis en avant c'est le travail de grandes associations, c'est l'événementiel, le maire l'indiquait véritablement: 'La seule industrie c'est le tourisme et ma politique culturelle ce sont les grands événements', c'est-à-dire que ce qui est mis en avant c'est Étonnants Voyageurs, festival du livre d'aventure, c'est Quai des Bulles, festival de la bande dessinée, ce sont [...] des associations qui sont soutenues à partir du moment où elles mettent en avant des actions ponctuelles, des actions qui apparaissent sur l'événementiel, c'est surtout ce qui apparaît dans la polémique malouine" (entretien avec le responsable du livre et de la lecture à la direction régionale des Affaires culturelles [DRAC], 31 mars 2008).

"La seule industrie, c'est le tourisme : deux millions de visiteurs ou plus à Saint-Malo par an, c'est énorme! Donc ça fait viure les hôtels, les crêperies, les restaurants, les cafés, les marchands de souvenirs, tout ça c'est une industrie qui tourne autour du tourisme. L'idée du maire [...], il l'a dit plusieurs fois : 'ma politique culturelle ce sont les grands événements'. Il ne s'en est pas caché, pourquoi ? Parce que si vous créez des grands événements vous attirez du monde" (entretien avec le conservateur des bibliothèques de SaintMalo, 21 avril 2007).

Jean-Claude Passeron distingue plusieurs fins de l'action culturelle, l'une d'elles s'intéresse à la proposition d'une "légitimité alternative" (Passeron, 1991, p. 292). Le festival de la bande dessinée Quai des Bulles s'inscrit dans cette promotion des "cultures populaires" et nous ne pouvons saisir cette légitimation qu'en la comprenant au prisme de la genèse de l'imposition d'une catégorie de pensée à l'échelle nationale. Le travail de représentation politique inauguré par le ministère d'André Malraux dans les années 1960, tendant à imposer la croyance selon laquelle un processus de "démocratisation culturelle" serait à l'œuvre, concourt à la mise en place de nouvelles structures que sont les maisons de la culture ${ }^{(15)}$. La conservation de cette vision se poursuit dans les années 1980 afin de contribuer à "l'élargissement de la légitimité artistique à des formes 'mineures' ou marginales” (Dubois, 1999, p. 283). Le champ de production idéologique - dans lequel s'inscrivent les acteurs du champ politique et du champ intellectuel - participe de la transformation des visions du monde, la bande dessinée n'étant pas considé- rée jusqu'alors comme une littérature légitime. La structuration et l'autonomisation du champ de la bande dessinée, observable au niveau national - notamment à travers la création de revues, d'académies, de sociétés savantes, de prix et d'événements (Boltanski, 1975) - ne peut se traduire localement qu'à la condition d'une admission dans les schèmes de perception et les comportements des agents sociaux de cette pratique culturelle reconnue comme légitime. Il s'agit de "refonder comme vie culturelle légitime des formes festives [...] qui sont déjà pratiquées et valorisées dans les groupes sociaux où s'investit [le] projet de revalorisation des cultures populaires ou régionales" (Passeron, 1991, p. 295). Le festival de la bande dessinée, caractérisé à ses débuts (1980) par ses pratiques amateurs, est créé à l'initiative d'un animateur ${ }^{(16)}$ de $\mathrm{La}$ Maison pour tous. L'association, alors imaginée dans une perspective d'accès de tous à la culture, ne dispose pas de trésorerie ni de structure d'accueil et l'invitation des dessinateurs repose essentiellement sur le capital relationnel constitué par le créateur du festival.

"On s'est dit comme ça, d'une façon tout ce qu'il y a de plus humble: [...] on pourrait faire un truc autour de la BD; c'est comme ça que ça a démarré [...] mais on ne savait pas trop comment s'y prendre: on contactait les auteurs et ils venaient mais sans vraiment savoir qui avait payé, qui sortait. C'était plus une ambiance comme ça, quoi, et la première année autour 
de ça s'est greffée une grosse fête, et les gars ont dit 'bah, faut pas arrêter, faut continuer parce que c'est avant tout notre fête'; c'était çà au départ, on se retrouve entre nous sans avoir toutes les contraintes" (entretien avec le créateur du festival Quai des Bulles, 15 mars 2008).

En 1990, Michel Le Bris organise une exposition à Saint-Malo sur l'écrivain Robert Louis Stevenson ; la rencontre avec le maire de SaintMalo, ajoutée à la fenêtre d'opportunité que représente l'idée de s'associer au festival Quai des Bulles, installé et reconnu depuis dix ans et bénéficiant d'un public stable, concourt à la création du festival Étonnants Voyageurs. Michel le Bris, né en 1944 à Plougasnou (Finistère), fils de femme de ménage et dépourvu de capital économique, grandit en Bretagne en ayant incorporé le rapport de domination propre à la relation qui s'exerçait entre "le maître" (Le Bris, 1977, p. 22) et sa famille et en ayant intériorisé les représentations véhiculées sur la Bretagne et les Bretons. Il appartient néanmoins, dans l'accumulation d'un capital culturel non pas hérité mais acquis par l'institution scolaire, à ces catégories de militants engagés dans les événements de mai-juin 1968. La dotation en capitaux, correspondant à des trajectoires déterminantes de positions à peu près équivalentes dans l'espace social, se trouve au point de rencontre "d'événements collectifs" dépendant euxmêmes de ces capitaux, de ces trajectoires, de ces positions (Bourdieu, 2007, p. 122 [1979]). Michel Le Bris détient ses ressources de son passage par l'école normale de Quimper, de ses études poursuivies aux lycées Hoche à Versailles et Louis-le-Grand à Paris puis à l'école HEC, et de l'obtention d'une maîtrise de philosophie. Le niveau de diplôme met en évidence la dotation en capitaux scolaires et, par là même, en capitaux culturels, déterminant la position occupée dans l'espace social ainsi que la capacité à investir ces capitaux symboliques dans les rapports de force engagés dans un espace donné - ces capitaux culturels étant euxmêmes convertis en un capital politique. Issu de ce monde intellectuel qui avait subi les désillusions propres à un contexte de remise en cause des institutions scolaires ne répondant plus aux aspirations professionnelles envisagées relativement à leur dotation en capitaux culturels et littéraires, Michel Le Bris investit ses ressources militantes dans $\mathrm{La}$ Cause du peuple (qu'il fonde et dirige avec Jean-Paul Sartre) et, comme de nombreux intellectuels engagés alors, reconvertit ses capitaux culturels dans le journalisme (Libération et L'Équipe) ; une forme de reclassement correspondant aux aspirations qui étaient celles d'une élite intellectuelle (Bourdieu, 2007 [1979]). L'entrée dans le champ littéraire s'opère pour Michel Le Bris par la reconnaissance de ses publications et de son action - comme responsable de la collection "Voyageurs" aux éditions Payot, créateur de la "Petite Bibliothèque Payot/ Voyageurs" et fondateur de la revue Gulliver en $1990^{(17)}$.
À travers l'organisation conjointe de ces deux éditions par les associations des festivals se structurent des rapports de force caractéristiques de façons de faire différenciées, ellesmêmes révélatrices de façons de penser en accord avec les représentations produites au sein du champ littéraire. Ainsi l'investissement d'un capital symbolique dans les luttes dépendil de la position de chaque agent dans le champ littéraire. Les rapports de force entre les deux festivals relèvent de la "distinction naturelle" appelant à la "distinction culturelle", qui consiste - afin de légitimer une pratique - à travailler à rendre les autres illégitimes (Bourdieu, 2007, p. 6 I, p. 254 [1979]). L'éclairage fait par les organisateurs d'Étonnants Voyageurs sur des pratiques considérées alors comme amateurs met en évidence la reproduction du rapport de domination institué au sein du champ littéraire. Le processus de professionnalisation, créateur d'une distance sociale entre une initiative culturelle visant un public local et des stratégies commerciales propres au public touristique, contribue dès lors au transfert de "l'art pur" vers "l'art commercial" (Bourdieu, 1994, p. 73). La professionnalisation du festival Étonnants Voyageurs, observable dans les transformations relatives à l'investissement et à la gestion financière ${ }^{(18)}$, le recrutement des professionnels et des bénévoles du festival ainsi que la structure du public visé (un public a priori "lettré") permettent d'entrevoir comme une fin possible de l'action culturelle, la conservation 
des visions dominantes sur la culture légitime et la consécration, en l'espèce, de la littérature légitime. La désignation des instances culturelles "légitimes" s'opère grâce aux institutions qui procèdent de cette légitimation, et notamment les institutions administratives et politiques. Les champs politique, intellectuel et artistique contribuent en ce sens à la confirmation d'un arbitraire. Ce qui passe dès lors pour des "besoins culturels" (Passeron, 1991, p. 338) dissimule - au travers du processus de professionnalisation de structures - des besoins économiques œuvrant à l'institutionnalisation d'une politique du livre et de la lecture.

\section{Des usages différenciés du livre dans la course à l'identité locale}

L'exemple des villes de SaintMalo, Bécherel et Dinan, appréhendées dans des contextes historiques et des configurations territoriales spécifiques et selon les rapports de force qui les structurent, permet de rendre compte des enjeux touristiques et politiques du livre ; "le livre" étant entendu dans toutes les acceptions que l'intervention d'une catégorie de l'action publique suppose au travers de la lecture publique, de l'édition et de la librairie, ici distinctes pour des raisons de présentation, interagissant néanmoins dans ce que d'aucuns appellent "la chaîne du livre". Le travail comparatif - envisagé dans une perspective relationnelle - nous éclaire sur la façon dont est menée une poli- tique culturelle. La proximité géographique des villes de Saint-Malo, Dinan et Bécherel les soumet à un principe de concurrence auquel se confrontent les collectivités locales, et dans lequel elles se confortent. Ainsi l'identité culturelle correspondant à chaque municipalité s'estelle nécessairement construite en fonction des autres. Agissant sur la base de modèles types d'action publique, les stratégies municipales - pour une finalité touristique qui peut sembler la même - n'en sont pas moins différentes dans leurs usages du livre. Baptisée "Cité du livre" en 1989, Bécherel est un "village de libraires" qui trouve son origine dans l'association Savenn Douar, lancée en 1986. Cette "petite cité de caractère" - dotée d'un patrimoine historique constitutif a priori d'une ressource culturelle aux enjeux politiques - s'inscrit dans un projet culturel et néanmoins économique d'identification territoriale. "Ville d'art et d'histoire" et "secteur sauvegardé de France", Dinan se distingue - dans la réalisation de sa politique culturelle et touristique par son usage différencié du patrimoine historique. La création d'une bibliothèque-médiathèque est le fruit de rapports de force engagés dès 1988 par le bibliothécaire de la ville de Dinan afin de voir naître des structures s'employant, dans les années 1990, à répondre à l'impératif culturel de démocratisation de la lecture (Bertrand, 2002).

"L'idée c'était un projet à caractère culturel et économique sur un territoire qui a une identité forte en
Bretagne et d'utiliser les atouts de ce territoire identitaire en tant que patrimoine, culture, histoire, pour implanter des activités économiques. [...] C'est la culture qui est la nouvelle économie c'est clair, la culture, le tourisme culturel" (entretien avec la fondatrice de la Cité du livre, 28 février 2008).

"Le parking souterrain favorise la visite du centre historique de Dinan; si vous venez à Dinan avec des amis, vous serez heureux aux mois de juillet et août sans doute de le trouver pour pouvoir être au cour de la ville et aller faire une visite, manger une crêpe, prendre un pot et voir les monuments, etc." (entretien avec le bibliothécaire de Dinan, 4 mars 2008).

L'appropriation de structures associatives par les pouvoirs locaux est un trait commun aux collectivités locales qui, dans la délégation de l'action publique par l'État, se sont inscrites dans des rapports de force les autorisant à se saisir d'objets culturels à des fins économiques. Le regard porté sur les variations constatées dans les différentes politiques du livre et de la lecture ne dispense pas de soulever dans l'analyse l'usage récurrent de l' "histoire" - entendue comme un ensemble de représentations et de pratiques associées à ces représentations, et qu'il convient d'historiciser dès lors que celle-ci prend les traits d'une histoire légitime et officielle renfermant en réalité la lutte pour sa naturalisation. Une politique tournée vers la librairie, une politique de lecture publique (avec tous les enjeux sociaux que 
celle-ci induit) ou encore une politique orientée vers des festivals inscrivent les collectivités et acteurs territoriaux dans une course à l'identité culturelle menant à bien la ou les politiques touristiques. "Cette quête du tourisme 'identitaire', niche marketing au nouvel avatar de l'illusion touristique qui peine à se matérialiser dans la demande actuelle, pourrait-elle être 'tirée' par une offre locale, véritable précurseur d'une différence porteuse de retombées économiques?” (Furt, 2007, p. 19). En d'autres termes, le tourisme estil un artefact qui nous empêche d'entrevoir les rapports de force qui se jouent entre les collectivités locales ? La lecture publique, la librairie et les festivals apparaissent dès lors comme le résultat d'une course à "l'identité" engagée afin de savoir laquelle de ces villes - par son attrait culturel vecteur de profits économiques - gagnera la compétition symbolique au sein de l'espace régional. Les différentes stratégies touristiques pour l'acquisition d'un capital de réputation d'une manifestation culturelle s'expliquent par un ensemble de variables que sont la structure du public, la saisonnalité ou le territoire ${ }^{(19)}$. Le lieu, ici "le littoral" - ou l'image qui lui est traditionnellement adjointe comme résultant des représentations et des modes de classification issus de la catégorie morale de tourisme et selon lesquels, "en s'appuyant sur de fortes évidences géographiques (le littoral, la montagne), on crée de la différence là où elle n'existe pas ou, du moins, là ou elle n'est pas nécessairement la plus porteuse de sens" (Stock, 2003, p. 17) -, signifierait davantage une fois associé à la saisonnalité. Il convient néanmoins d'appréhender ces variables au regard des trajectoires particulières des acteurs locaux, positionnés dans l'espace social selon les rapports de force (entre les champs associatif et politique notamment) qui les hiérarchisent, et en fonction des conditions historiques régionales et nationales de formation d'une politique publique.

La production d'une "image" apparaît dès lors comme une construction sociale, un travail d'homogénéisation qui conduit à s'interroger sur les producteurs de ces représentations. Les acteurs du champ politique local, les acteurs du monde de l'édition, du champ littéraire mais également médiatique participent conjointement de l'attraction touristique. Toutefois, la contribution des médias aux visions légitimement instituées consiste davantage à produire des différences entre les politiques du livre, et à favoriser en ce sens la pérennité des manifestations culturelles, qu'à "retenir le visiteur d'un jour" (Bourdieu et Darbel, 1969, p. 130). L'intervention des différents médias est déterminée par les capitaux relationnels détenus par les organisateurs des manifestations culturelles (notamment Michel Le Bris et le réseau journalistique important dont il dispose, du fait de sa trajectoire particulière) ainsi que par les acteurs du champ politique ${ }^{20)}$. Ces "incitations directes" que sont les articles de presse, les émissions radiophoniques et télévisées, ou encore "les panneaux apposés à l'entrée des villes touristiques" (Bourdieu et Darbel, 1969, p. 146) l'on pense notamment au label Ville d'art et d'histoire - ne font pas davantage se déplacer les exclus de l'offre culturelle. C'est dire que la consommation culturelle est contrainte, d'une part, par les conditions économiques du tourisme (la possibilité matérielle de voyager) et, d'autre part, par les déterminations sociales induites par toute offre culturelle. La dotation en capitaux économiques - relative au niveau de revenus et observable dans le prix d'entrée du festival (10 euros pour Étonnants Voyageurs) ${ }^{(21)}$, le coût du voyage, du logement et de la restauration - ainsi qu'en capitaux culturels influent par conséquent sur la structure du public. Les caractéristiques sociales déterminent les prédispositions à fréquenter la manifestation, elles-mêmes conditionnées par les représentations que l'on se fait de celle-ci, de son contenu, de son public a priori "lettré" ou non (l'on pense notamment à Étonnants Voyageurs) - l'institutionnalisation d'un événement culturel tenant à un ensemble de croyances et de pratiques qui permettent sa pérennité. Par ailleurs, l'action opérée par les producteurs de représentations n'est réalisée que si elle est confirmée par le système scolaire qui favorise la reproduction de façons de faire et de penser. Les journées scolaires, organisées par la ville de Saint-Malo pour le festival Quai des Bulles ou par la ville de Dinan à la biblio- 
thèque, contribuent en ce sens à favoriser l'accès des classes sociales défavorisées à la culture.

L'initiative culturelle constitue un enjeu politique dès lors qu'elle est saisie et réappropriée par les professionnels de la politique. "Le livre" en est l'illustration, en ce sens qu'il participe dans ses visions et ses usages différenciés, pour un ensemble d'acteurs hétérogènes, à la conservation ou à la transformation d'une image culturelle de la région. Au risque de défaire certaines représentations spontanées constituées autour de la valeur effective du tourisme comme agissant seul sur la pratique culturelle, il convient d'envisager celui-ci non plus comme une ressource politique, mais davantage comme un élément de la politique du livre et de la lecture. "Le tourisme, capable de favoriser la pratique, ne peut par soi (toutes choses égales d'ailleurs) créer une pratique durable et modifier durablement les probabilités et les taux de fréquentation” (Bourdieu et Darbel, 1969, p. | |6-| |7). Le tourisme, partie prenante de la politique culturelle de la région, confirme les prédispositions à assister aux manifestations culturelles et accentue en ce sens la pratique qui s'y attache.
NOTES

(I) CONSEIL ÉCONOMIQUE, SOCIAL ET ENVIRONNEMENTAL RÉGIONAL DE BRETAGNE, Le

Tourisme en Bretagne. $4^{\mathrm{e}}$ réunion ordinaire, novembre 1993, p. 7.

(2) En référence à la définition de

l'Organisation mondiale du tourisme (OMT) évoquée dans l'ouvrage.

(3) Cf. L'Impératif culturel, rapport du commissariat général au plan, novembre 1982.

(4) Les aménagements touristiques seront observés à travers l'exemple d'usages différenciés du "livre" dans la mise en œuvre de politiques publiques envisagées relativement à un contexte particulier de formation de ces politiques publiques.

(5) Le "droit à l'autodétermination" est évoqué, dans les années 1980, comme le "droit à la différence", en référence au discours prononcé par François Mitterrand à

Lorient, le 14 mars 1981. "C'est blesser un peuple au plus profond de lui-même que de l'atteindre dans sa culture et sa langue. Nous proclamons le droit à la différence" (cf. Patez, 2001, p. 36).

(6) 'Le culte de la 'culture populaire' n'est, bien souvent, qu'une inversion verbale et sans effet, donc faussement révolutionnaire, du racisme de classe qui réduit les pratiques populaires à la barbarie ou à la vulgarité, [. . ] cette manière en définitive très confortable de respecter le 'peuple', qui, sous apparence de l'exalter, contribue à l'enfermer ou à l'enfoncer dans ce qu'il est [...] tout en laissant les choses en l'état, les uns avec leur culture (ou leur langue) réellement cultivée et capable d'absorber sa propre subversion distinguée, les autres avec leur culture ou leur langue dépourvues de toute valeur sociale ou sujettes à de brutales dévaluations [...] que I'on réhabilite fictivement par un simple faux en écriture théorique" (Bourdieu, 2003 [ [ ${ }^{\text {re }}$ édition 1997], pp. | 10- | | |).
(7) "Le fait fondamental de cette charte est de reconnaître non seulement l'existence de la personnalité culturelle de la Bretagne, mais encore de permettre à cette personnalité de se maintenir et de s'affirmer" (extrait du discours de Valéry Giscard d'Estaing, le 8 février 1977 à Ploërmel ; cf. "Signature de la Charte culturelle de Bretagne. Régions : une révolution tranquille", Le Figaro,

I I février 1978).

(8) Archives du conseil régional de Bretagne, 93WI63, "Charte culturelle de Bretagne. Bilan au I er novembre 198I".

(9) Les études sociologiques menées sur la question rendent compte de déterminants socio-professionnels de l'accès à la position d'élu (Offerlé, 2008, p. 79).

(I0) CONSEIL ÉCONOMIQUe, SOCIAL ET ENVIRONNEMENTAL RÉGIONAL DE BRETAGNE, op. cit., p. 7.

(I I) Ne remplissant plus les conditions, la ville de Saint-Malo a aujourd'hui perdu son label ; cependant la pancarte "Ville d'art et d'histoire" reste affichée à l'entrée de la ville.

(I2) Le Conseil culturel de Bretagne et I'Institut culturel de Bretagne (ICB), qui regroupent des acteurs de la vie culturelle bretonne. Le Comité d'études et de liaison des intérêts bretons (CELIB) lance l'idée d'un institut culturel en 195I. L'ICB, issu de la Charte culturelle de Bretagne de 1978, est créé en 1981.

(13) Archives du conseil régional de Bretagne, dossier: "Centre régional du livre en Bretagne".

(14) Entre 180000 et 220000 euros, selon l'organisateur du festival Étonnants Voyageurs (entretien avec l'organisateur, 7 avril 2007).

(I5) Les maisons de la culture sont créées dans une "volonté de diffuser largement la jouissance des œuvres savantes ou lettrées" (Passeron, 1991, p. 295). Elles étaient cen- 
sées inciter les classes moyennes et populaires à la pratique culturelle. Toutefois, "loin de remplir la fonction qu'une certaine mystique de la culture populaire lui assigne, la maison de la culture reste la maison des hommes cultivés" (Bourdieu et Darbel, 1969, p. 150).

(16) Profession de porte-parole caractéristique des "nouvelles fractions des classes moyennes, détentrices de capital culturel mais dominées dans l'ordre des hiérarchies intellectuelles et sociales" (Boltanski, 1975, p. 46). (17) La revue Gulliver a été créée en vue de rééditer les œuvres de Robert Louis Stevenson

( 18 ) Les subventions allouées à Étonnants Voyageurs sont passées de 80798 euros en 1995 à 326850 euros en 2005 (source : archives municipales de la ville de SaintMalo). Les chiffres de 1990 à 1994 ne sont pas communiqués par la ville.

(19) La structure du public varierait selon le moment (la "saison") et l'espace géographique de l'offre culturelle (Négrier, 2010).

(20) Voir notamment le travail de Patrick Champagne sur la question des transferts entre le champ médiatique et le champ politique (Champagne, 1990).

(2I) "Les classes supérieures sont davantage présentes lors des spectacles les plus chers, là où les classes populaires et moyennes obtiennent leur plus faible score. [...] Le prix constitue bien un frein à la participation culturelle d'une manière générale, et notamment chez les classes populaires" (Négrier, 2010, p. 159).

\section{RÉFÉRENCES BIBLIOGRAPHIQUES}

Paul Aluı̇̇s, "Territoire régional et représentation des intérêts", Politix, vol. 2, n०7-8, 1989, pp. 63-71.

Lionel Arnaud, Christian Le BART et Romain PASQUier, Idéologies et action publique territoriale. La politique change-t-elle encore les politiques?. Presses universitaires de Rennes, 2006.

Philippe BACHIMON, "Tourisme et identité. Un rapport ambigu", dans JeanMarie FURT et Franck MICHEL (dir.), Tourismes et identités, L'Harmattan, 2006. Jean-François BAYART, L'Illusion identitaire, Fayard, 1996

Catherine BERTHO, "L'invention de la Bretagne. Genèse sociale d'un stéréotype", Actes de la recherche en sciences sociales, n³, novembre 1980, pp. $45-62$.

Anne-Marie BerTRAND, Les Bibliothèques municipales : enjeux culturels, sociaux, politiques, Cercle de la Librairie, 2002.

Luc BoltANSKI, "La constitution du champ de la bande dessinée", Actes de la recherche en sciences sociales, vol. I, $n^{\circ}$ I, 1975, pp. 37-59.

Pierre BOURDIEU, "L'identité et la représentation", Actes de la recherche en sciences sociales, vol. 35, $n^{\circ}$ I, 1980, pp. 63-72.

Pierre Bourdieu, Raisons pratiques. Sur la théorie de l'action, Seuil, 1994.

Pierre BouRdieu, Méditations pascaliennes, Seuil, 2003 (I édition 1997).

Pierre Bourdieu, La Distinction. Critique sociale du jugement, Éditions de Minuit, 2007 (I éédition 1979).

Pierre Bourdieu et Alain DARBEL, L'Amour de l'art. Les musées d'art européens et leur public, Éditions de Minuit, 1969.

Pierre BOURDIEU, Roger ChARTIER et Robert DARNTON, "Dialogue à propos de l'histoire culturelle", Actes de la Recherche en sciences sociales, vol. 59 , $n^{\circ}$ I, 1985, pp. 56-93.

Philippe BRAUD, Sociologie politique, LGDJ, 2008

Patrick Champagne, Faire l'opinion. Le nouveau jeu politique, Éditions de Minuit, 1990.

Dominique Damamme, Boris GoBILLE, Frédérique MATONTI et Bernard

PudAL, Mai-Juin 68, Éditions de l'Atelier, 2008.

Pascal DAUvin, Constructions et représentations de l'institution régionale. Analyse d'un processus inachevé. L'exemple breton, thèse de doctorat en science politique, Université Rennes I, 1993.

Vincent DuBoIs, La Politique culturelle. Genèse d'une catégorie d'intervention publique, Belin, 1999.

Yann FourNIs, Les Régionalismes en Bretagne ; la région et l'État (1950-2000), Presses interuniversitaires européennes, 2006. 
Jean-Marie FURT, "Vendre l'identité d'une destination", dans Jean-Marie FURT et Franck MiCHeL (dir.), L'Identité au cœur du voyage, L'Harmattan, 2007.

Patrick HASSENTEUfel et Andy SMITH, "Essoufflement ou second souffle?

L'analyse des politiques publiques 'à la française"', Revue française de science politique, vol. 52, n I, 2002, pp. 53-73.

Michel Le BRIS, L'Homme aux semelles de vent, Payot, 1977.

Emmanuel NÉGrIer (dir.), Les Publics des festivals, Michel de Maule - France festivals et Réseau en scène Languedoc-Roussillon, 2010.

Michel Offerlé, Les Partis politiques, Puf, 2008.

Romain PASQUIER, La Capacité politique des régions : une comparaison France/Espagne, Presses universitaires de Rennes, 2004.

Jean-Claude PASSERON, Le Raisonnement sociologique. L'espace non-poppérien du raisonnement naturel, Nathan, 1991.

Fabrice PATEZ, "Réflexions sur la notion d'identité culturelle dans les mouvements régionaux français", Les Cahiers du Cériem (Centre d'étude et de recherche sur les relations Inter-Ethniques et les minorités), nº 7, juin 200 I, pp. $33-42$.

Pierre-Jean Simon, La Bretonnité. Une ethnicité problématique, Presses universitaires de Rennes, 1999.

STENDHAL, Mémoires d'un touriste en Bretagne, Éditions Entente, 1984 (récit paru en 1838).

Anne-Marie THIESSE, La Création des identités nationales : Europe, XVIII'-XXe siècle, Seuil, 1999.

Mathis Stock (coord.), Le Tourisme. Acteurs, lieux et enjeux, Belin, 2003. Eugen WEBER, La Fin des terroirs. La modernisation de la France rurale : I87019/4, Fayard, 1983. (Traduit de l'anglais par Antoine BERMAN et Bernard GÉNIÈs.)

\section{ARCHIVES ET RÉFÉRENCES INSTITUTIONNELLES}

ARChives municipales DE LA VILLE DE SAINT-MaLo (Subventions associations 1990-1992, 1994-2005).

ARCHIVES DU CONSEIL RÉGIONAL DE BRETAGNE (dossier 93WI 63 ; dossier : "Centre régional du livre en Bretagne" ; Le Figaro, II février 1978).

CONSEIL ÉCONOMIQUE, SOCIAL ET ENVIRONNEMENTAL RÉGIONAL (CESER) RÉGION BRETAGNE, Le Tourisme en Bretagne. 4e réunion ordinaire, novembre 1993. 